\title{
Vacuum Sampling in the Landscape during Inflation
}

\author{
Hooman Davoudiasl, ${ }^{1,2}$ Saswat Sarangi, ${ }^{3}$ and Gary Shiu ${ }^{2}$ \\ ${ }^{1}$ Department of Physics, Brookhaven National Laboratory, Upton, New York 11973-5000, USA \\ ${ }^{2}$ Department of Physics, University of Wisconsin, Madison, Wisconsin 53706, USA \\ ${ }^{3}$ Department of Physics, Columbia University, New York, New York 10027, USA
}

(Received 24 May 2007; published 18 October 2007)

\begin{abstract}
We consider the phenomenological consequences of sampling multiple vacua during inflation motivated by an enormous landscape. A generic consequence of this sampling is the formation of domain walls, characterized by the scale $\mu$ of the barriers that partition the accessed vacua. We find that the success of big bang nucleosynthesis (BBN) implies $\mu \gtrsim 10 \mathrm{TeV}$, as long as the sampled vacua have a nondegeneracy larger than $\mathcal{O}\left(\mathrm{MeV}^{4}\right)$. Otherwise, the walls will dominate and eventually form black holes that must reheat the universe sufficiently for BBN to take place; in this case, we obtain $\mu \gtrsim 10^{-5} M_{P}$. These black holes are not allowed to survive and contribute to cosmic dark matter density.
\end{abstract}

PACS numbers: $98.80 . \mathrm{Cq}$

Various theoretical considerations have led to a picture of string theory with a gigantic number of solutions, referred to as the "landscape" of vacua [1]. This picture was motivated in part by the construction of cosmological vacua in Refs. [2,3], and the dense "discretuum" in Refs. $[4,5]$. To confront the vast landscape of string vacua, a statistical approach has also been proposed [6]. Despite progress, a detailed understanding of the topography of the landscape remains elusive.

Usually, effects due to the string theory landscape are relegated to eternal inflation. Once the universe enters the last stage of inflation (that includes the last $60 e$ folds), the multiple vacua cease to have any effect, and the universe classically evolves towards a single vacuum in the landscape. Portions of the universe in different vacua are exponentially far away from each other and the landscape seemingly does not leave any imprints on the postinflationary cosmology; see, e.g., Ref. [7].

Since inflation is well motivated and strongly supported by data, we assume that it is part of cosmic history. Inflation is generically characterized by enormous energy scales, at which other vacua may be accessed. An enormous landscape can then be expected to affect cosmic evolution. In this Letter, we consider the general possibility that the fundamental description of nature entails a large landscape of vacua, some of which may be accessible during the last $60 e$ folds of inflation. We then inquire how this premise affects cosmological evolution.

Inflationary vacuum sampling may proceed in various ways. For example, during inflation, characterized by a Hubble constant $H$, a light field $S$ of mass $m_{S} \lesssim H$ develops de Sitter fluctuations $\delta S \sim H$ and may sample the local landscape. In many situations, as in minimal models of supersymmetric inflation, there are noninflaton fields that are light during inflation with masses a few orders of magnitude below $H$. Such fields and their cosmological effects have been studied, e.g., in Refs. [7,8]. If initially $S$ is in one of the minima, given a modest number of $e$ folds, the field can get delocalized and make excursions to other vacua, for example, because: (i) the neighboring vacua are separated by shallow barriers, or (ii) due to resonance effects, tunneling transitions between certain vacua take place unsuppressed [9].

A demonstration of the relative likelihood of the above, or other, mechanisms for vacuum sampling requires a detailed knowledge of how the landscape topography is generated microscopically and is not within the scope of this work. However, it has been argued that a large number $\mathcal{N} \geq 10^{120}$ of vacua may be necessary to accommodate a cosmological constant $(\mathrm{CC})$ of order $\left(10^{-30} M_{P}\right)^{4}$, as inferred by the size of dark energy density. In fact, the success of Weinberg's prediction [10] for the approximate order of magnitude of the $\mathrm{CC}$ may point to a more dramatic possibility that our local region of the landscape is a dense attractor [11-13]. Similar attractor behavior also seems to appear in the landscape of flux vacua [14-16]. Given such a large number of vacua, one may expect that a few local directions will be accessible to vacuum sampling during inflation. Hence, we take the possibility of more than one vacuum being accessed during inflation to be a plausible assumption and will study its phenomenological consequences.

We expect vaccum sampling to be important only during inflation. For example, if sampling is mediated by the above mentioned light scalar $S$, after inflation, this field no longer has de Sitter fluctuations and settles down to one vacuum. Quite generally, we expect each causal patch of size $H^{-1}$ to settle into a different accessible vacuum (assuming a flat slicing of de Sitter spacetime at the end of inflation when the walls form as the field settles into different minima). At the end of inflation and henceforth, we expect each patch will follow the usual single-vacuum evolution. However, different causal patches will be separated by domain walls. The future evolution will depend on whether the walls percolate or not. This depends on the relative population of each vacua, i.e., the number of 
Hubble patches that end up in different vacua. For two vacua, the relative population in each vacuum should be above 0.31 for percolation to happen [17]. Presumably, the relative population will depend on the split in the degeneracy $\epsilon^{4}$ between the vacua. For example, for the case where the sampling of vacua happens due to Hubble fluctuations allowing the field to hop over small barriers, the ratio of populations will go as $\exp (-\epsilon / H)$. Note that $\epsilon$ is bounded by the height of the barrier, which for sampling to work, should be of the order $H$ or less. For $\epsilon$ an order of magnitude below $H$, the relative populations easily satisfy the condition for percolation. If the walls percolate, the universe will consist of a long infinite wall of a complicated geometry stretching across the three-dimensional space, and there will be a distribution of finite clusters of walls. For $\epsilon$ close to $H$ percolation might not happen. If the walls do not percolate, then there will just be clusters (bags of domain walls enclosing one type of vacuum within and the other type of vacuum outside) of various sizes. The clusters should go away by the time of big bang nucleosynthesis (BBN).

From now on we consider the case in which the domains percolate and horizon-sized domain walls form. The tension of these domain walls depends on the moduli, set by the local topography of the landscape. These walls can in principle lead to serious problems for cosmology [18]. First of all, they can quickly come to dominate the energy density of the universe and disrupt radiation domination that must set in, say, by the time of the BBN. Second, if the walls exist after recombination, unless their tension is below $(\mathrm{MeV})^{3}$, they would lead to large anisotropies in the cosmic microwave background radiation.

Therefore, it seems that our picture has led to an unacceptable state for the early universe. However, this is not necessarily the case. Domain walls are stable only if they come from spontaneously broken discrete symmetries. In that case, the various domains are degenerate vacua of the theory. During vacuum sampling of the landscape, however, there is no symmetry that would require different causal patches to have the same vacuum energy. If the vacua are not degenerate, as is well known [19,20], the unwanted walls can be pushed away into the false vacuum regions, by the pressure from the energetic bias, resulting in a universe dominated by the true vacuum. We will next study the phenomenological constraints that these considerations may impose on the local landscape.

The above solution to the domain-wall problem is usually based on a scenario in which the universe is partitioned into two phases by a typically complicated domain wall. The two-phase universe is a result of percolation, a process that depends on the relative probability for each phase to emerge in a given causal patch. The situation resulting from vacuum sampling of the landscape could, in principle, be more complicated. It is a possibility that a sufficient number of local minima might be accessible so that the domain walls are approximately (as these symmetries are not exact) of the type $Z_{N}$, with $N>2 . Z_{N}$ domain walls typically consist of configurations of $N$ domain walls attached to a string [21].

To have a more transparent analysis, let us consider the simple case of only two accessible vacua at the end of inflation. This yields two dominant vacuum populations, one of which leads to our universe. Our treatment follows considerations similar to those in Ref. [22]. Wall formation happens at the end of inflation (as access to multiple vacua is lost), at time $t_{\mathrm{wf}} \simeq t_{I} \sim 1 / H$, where the subscript $I$ denotes the end of inflation. Here, $H \sim M_{I}^{2} / M_{P}$, where $M_{I}$ is the is the inflationary scale, and the $M_{P}$ is the 4- $d$ Planck scale. We will assume that the wall is flat over a causal patch. Thus, the energy density in the wall, characterized by a scale $\mu$, is given by $\rho_{w} \sim \mu^{3} / t$. $t$ is given by the size of the causal patch. The radiation energy density is then given by $\rho_{r} \sim M_{P}^{2} / t^{2}$. We take the universe to be initially radiation dominated, which is a typical assumption [19]. Domain-wall domination $\left(\rho_{w}>\rho_{r}\right)$ begins at a time scale

$$
t_{\mathrm{wd}} \sim M_{P}^{2} / \mu^{3} .
$$

Until time $t_{\mathrm{wd}}$, the evolution of the postinflationary universe is governed by radiation domination; $a \propto t^{1 / 2}$, where the scale factor is denoted by $a$.

Once the domain walls form, they can lead to two alternative cosmologically viable scenarios. The first scenario requires that vacuum states separated by walls do not have degenerate energy densities. As we discussed before, if $\epsilon \sim H$, percolation might not happen, and then, as we shall show, the clusters of domain walls will disappear automatically. We now consider the case where $\epsilon$ is small compared to $H$ such that percolation does happen. As a toy model of the landscape we consider just two vacua with an energy density difference $\epsilon^{4}$. Here, we require that the walls disappear before they dominate the universe. This can happen if $\epsilon>\epsilon_{*}$ which we shall now determine. The bias $\epsilon$ becomes important at time $t_{\epsilon}$ when the volume energy in the domain wall is of the order of the surface energy:

$$
t_{\epsilon} \sim \mu^{3} / \epsilon^{4} .
$$

For $\epsilon \neq 0$ the lowest energy vacuum prevails and the walls disappear. Requiring $t_{\epsilon}<t_{\mathrm{wd}}$, we get

$$
\epsilon_{*}^{4} \sim \mu^{6} / M_{P}^{2}
$$

However, we still do not want the walls to disrupt wellestablished cosmological processes. Thus, we conservatively require that the walls disappear before BBN: $t_{\mathrm{wd}}<$ $t_{\mathrm{BBN}}$. This yields $\mu \gtrsim 10 \mathrm{TeV} ; \epsilon_{*}^{4} \gtrsim \mathrm{MeV}^{4}$.

If $\epsilon<\epsilon_{*}$, we have the second scenario. Note that string theory generically allows for axionic potentials where $\epsilon=$ 0 and $\mu$ is finite [23]. The universe becomes domain-wall dominated at $t \sim t_{\mathrm{wd}}$ and the domain walls grow till there is 
a gravitational instability that makes them collapse into black holes. In fact, the time at which the wall collapses to black holes coincides with $t_{\mathrm{wd}}$, i.e., $t_{\text {collapse }}=t_{\mathrm{wd}}$. To see this, let us first estimate the mass of a black hole thus formed. Consider a sphere of growing radius $R$, centered on the wall. The mass of the wall inside the sphere is given by $M(R) \sim R^{2} \mu^{3}$. (We assume that the energy due to the bias $\epsilon$ at such radius $R$ is less than that due to the wall tension. This is a justified assumption since $\epsilon<\epsilon_{*}$ and the collapse happens before the volume term dominates.) When $R=R_{\mathrm{bh}} \sim M_{P}^{2} / \mu^{3}$, the condition $M_{P}^{-2} M(R)=R$ will be satisfied and the wall will collapse into a black hole of mass

$$
M_{\mathrm{bh}} \sim\left(M_{P} / \mu\right)^{3} M_{P} .
$$

Now if we use the time dependence of $R, R \sim t$, then we see that the time at which the wall collapses to black holes is given by $t_{\text {collapse }} \sim M_{P}^{2} / \mu^{3}$, coinciding with $t_{\mathrm{wd}}$. When the wall is about to become the dominant form of energy, it collapses into black holes. The universe will now become black hole dominated. However, depending on how light these black holes are, they can quickly evaporate. The time scale for black hole evaporation is

$$
\tau_{\mathrm{bh}} \sim M_{\mathrm{bh}}^{3} / M_{P}^{4} \text {. }
$$

If the wall tension is set by $\mu \sim 10^{-3} M_{P}$, which can be a reasonable string scale $M_{s}$, then $\tau_{\text {bh }} \sim 10^{-16} \mathrm{~s}$.

An interesting outcome of the above process is that black hole evaporation can reheat the universe to high temperatures again. Thus, vacuum sampling may lead to landscape-assisted reheating. To see this, we note that at formation, the energy density in black holes is

$$
\rho_{\mathrm{bh}}\left(t_{\mathrm{wd}}\right) \sim \mu^{6} / M_{P}^{2}
$$

After the collapse of the walls into black holes at $t_{\mathrm{wd}}$, the evolution of the scale factor is that of a matter-dominated universe. Therefore, at time $\tau_{\mathrm{bh}}$, we have

$$
\frac{a_{\tau}}{a_{\mathrm{wd}}}=\left(\frac{\tau_{\mathrm{bh}}}{t_{\mathrm{wd}}}\right)^{2 / 3} \sim\left(\frac{M_{P}}{\mu}\right)^{4} .
$$

Thus, the energy density in black holes just before their decay, approximated as instantaneous, is

$$
\rho_{\text {bh }}\left(\tau_{\text {bh }}\right) \sim \mu^{18} / M_{P}^{14} .
$$

If $\rho_{\mathrm{bh}}\left(\tau_{\mathrm{bh}}\right)$ is converted into radiation at $t \sim \tau_{\mathrm{bh}}$, the resulting temperature of the universe is

$$
T_{\mathrm{rh}} \sim\left[\rho_{\mathrm{bh}}\left(\tau_{\mathrm{bh}}\right)\right]^{1 / 4} .
$$

For $\mu=10^{-3} M_{P}$, as before, we get $T_{\mathrm{rh}} \sim 100 \mathrm{TeV}$, far above the weak scale, which is an acceptable reheat temperature. $T_{\mathrm{rh}}$ should be at least of order $1 \mathrm{MeV}$ to allow for the BBN. Hence, we conclude that to exit black hole domination gracefully, we must demand $\mu>\mu_{*} \sim$ $10^{-5} M_{P}$. Even if reheating can be achieved by the inflaton instead of black holes, for $\mu$ below this bound, the universe would not have exited the black hole domination phase quickly enough to allow for standard BBN.

One could envisage a situation where there is a distribution in $M_{\mathrm{bh}}$ such that some black holes might survive till today. These black holes essentially provide bridges to neighboring minima of the landscape while they exist. The spectrum of $M_{\mathrm{bh}}$ will depend on the detailed structure of the local string landscape. Depending on the spread in the heights of the barriers between neighboring minima, there will be a spread in $M_{\mathrm{bh}}$. However, as we shall see now, even with a mass distribution, all black holes should evaporate by reheating. That is to say, the heaviest black hole population (resulting from the collapse of the walls with the least tension) must be responsible for postinflationary reheating.

To demonstrate this point, let us consider a bimodal distribution of wall tensions with walls of type $A$ with mass scale $\mu_{A}$ and wall of type $B$ with tension $\mu_{B}$. We would like wall $A$ to reheat the universe via the evaporation of the black holes it would collapse to. So the previous analysis regarding black hole assisted reheat applies to wall $A$. The reheat energy is given by $\rho_{\text {reheat }} \sim \mu_{A}^{18} / M_{P}^{14}$. Now we would like to see if the black holes formed due to the collapse of the wall $B$ can be entirely responsible for dark matter. For these black holes to have survived till today, we must have $\mu_{B} \ll \mu_{A}$. This justifies the neglect of wall $B$ when we derive the reheat scale. The time of collapse of wall $B$ into black hole $B$ will be $t_{\text {collapse }}^{B} \sim M_{P}^{2} / \mu_{B}^{3}$. However, just as for wall $A$, the $t_{\text {collapse }}^{B}$ will coincide with the time when wall $B$ begins to be the dominant form of energy in the universe.

To see this, note that at all stages of the cosmological evolution, the energy density is redshifting as $1 / t^{2}$. First there is a radiation dominated stage, followed by a black hole $A$ dominated stage, followed by another radiation dominated stage due to reheat, and then at some time wall $B$ begins to dominate. The condition for wall $B$ domination is still $M_{P}^{2} / t^{2} \sim \mu_{B}^{3} / t$, i.e., $t_{\mathrm{wd}}^{B} \sim M_{P}^{2} / \mu_{B}^{3}$.

Hence, the time at which the energy density begins to be the dominant energy contribution exactly coincides with the time at which wall $B$ collapses to black hole $B$ and becomes the dominant component. Since we would like to use black hole $B$ as dark matter, this time must be the time of radiation-matter equality, at $T_{\text {eq }} \sim 1 \mathrm{eV}$ :

$$
t_{\mathrm{wd}}^{B} \sim t_{\text {collapse }}^{B} \sim M_{P}^{2} / \mu_{B}^{3} \sim M_{P} / T_{\mathrm{eq}}^{2} .
$$

This fixes the value of the mass scale of wall $B, \mu_{B} \sim$ $1 \mathrm{GeV}$. The mass of the resulting black holes is $\left(M_{P} / \mu_{B}\right)^{3} M_{P} \sim 10^{19} M_{\odot}$, where $M_{\odot} \sim 10^{30} \mathrm{~kg}$ is the solar mass. Such black holes are too heavy to make dark matter, since a typical galactic mass is of order $10^{11} M_{\odot}$.

Incidentally, this leads to a no-go theorem for walls that form around inflation to give dark matter via collapse into black holes. Let us consider $k$ different black holes with different tensions. We would like the last surviving black 
holes to give dark matter. All the previous black holes just release energy into radiation as they evaporate. However, as we have seen above, the time of the collapse of a wall into black holes exactly coincides with the time when the wall begins to be the dominant form of energy. This time is given by $t \sim M_{P}^{2} / \mu_{k}^{3}$. However, since we want these black holes to be dark matter, $t \sim M_{P} / T_{\mathrm{eq}}^{2}$, the time of radiationmatter equality. This fixes the mass of the black holes to be $10^{19} M_{\odot}$. Hence, we have a no-go theorem: Walls that form from inflationary vacuum sampling cannot yield black hole dark matter.

The minimum bias $\epsilon_{*} \sim \mathrm{MeV}$ we have derived suggests that unless the sampled vacua have large vacuum energies compared to that in our observable universe, we end up in a black hole dominated universe. In this case, the walls must be predominantly characterized by a scale larger than $\mu_{*} \sim 10^{-5} M_{P}$. Put another way, either none of the vacua in our local landscape are accessible to inflationary sampling or else all the accessible ones have large cosmological constants or high barriers. The above bounds are at best rough estimates and subject to well-defined assumptions. However, because the basic ingredients leading to these conclusions are general relativity, inflation, and quantum mechanics, the above considerations point to possible bottom-up constraints on the features of the local landscape. We note that even in the first scenario, where wall domination is avoided by having $\epsilon>\epsilon_{*}$, we derived a lower bound on the wall tension $\mu \geqslant 10 \mathrm{TeV}$ by requiring that the walls be gone before BBN, as a minimal normalcy condition.

In summary, we assumed that one or more efficient ways of transit to other landscape minima exist and access to a few vacua during inflation may well be plausible. This assumption is motivated by the large number of possible neighboring vacua and the closeness of inflationary and string scales. We examined domain-wall formation, as a generic consequence of vacuum sampling during inflation. The false vacua can be pushed out given a bias between the sampled vacuum energies. BBN considerations point to a minimum bias of order $\mathrm{MeV}^{4}$. The barriers are then characterized by a minimum scale of order $10 \mathrm{TeV}$. In the absence of the necessary vacuum energy bias, domainwall domination will ensue and lead to the genesis of primordial black holes. The evaporation of these black holes must reheat the universe. BBN constraints then suggest a minimum barrier scale of order $10^{-5} M_{P}$. We showed that these primordial black holes cannot survive to be part of cosmic dark matter. Our bottom-up approach could be viewed as a phenomenological guide for future landscape model building.

We thank X. Chen, P. Corasaniti, B. Greene, D. Kabat, J. Polchinski, and H. Tye for discussions. S. S. thanks the particle theory group at University of Wisconsin, Madison, and the Perimeter Institute. This work was supported in part by the DOE under Contracts No. DE-AC02-98-CH10886 (H. D.), No. DE-FG02-95ER40896 (H. D., G. S.), and No. DE-FG02-92ER40699 (S.S.), NSF Grant No. PHY-0348093 (G.S.), two grants from Research Corporation (G.S.), and a grant from the Department of Physics at the University of Wisconsin-Madison (H. D.).

[1] L. Susskind, arXiv:hep-th/0302219.

[2] S. Kachru, R. Kallosh, A. Linde, and S. P. Trivedi, Phys. Rev. D 68, 046005 (2003).

[3] S. Kachru, R. Kallosh, A. Linde, J. Maldacena, L. McAllister, and S.P. Trivedi, J. Cosmol. Astropart. Phys. 10 (2003) 013.

[4] R. Bousso and J. Polchinski, J. High Energy Phys. 06 (2000) 006.

[5] J.L. Feng, J. March-Russell, S. Sethi, and F. Wilczek, Nucl. Phys. B602, 307 (2001).

[6] F. Denef and M. R. Douglas, J. High Energy Phys. 05 (2004) 072; M. R. Douglas, C.R. Physique 5, 965 (2004); M.R. Douglas, Proceedings of the 2nd International Conference on String Phenomenology, Durham, UK, 2003 (World Scientific, Singapore, 2004).

[7] A. Linde and V. Mukhanov, J. Cosmol. Astropart. Phys. 04 (2006) 009.

[8] G. Dvali, A. Gruzinov, and M. Zaldarriaga, Phys. Rev. D 69, 023505 (2004).

[9] S.H. Tye, arXiv:hep-th/0611148.

[10] S. Weinberg, Phys. Rev. Lett. 59, 2607 (1987).

[11] A. D. Sakharov, Sov. Phys. JETP 60, 214 (1984) [Zh. Eksp. Teor. Fiz. 87, 375 (1984)]; Sov. Phys. Usp. 34, 409 (1991).

[12] G. Dvali and A. Vilenkin, Phys. Rev. D 70, 063501 (2004).

[13] G. Dvali, Phys. Rev. D 74, 025018 (2006).

[14] S. Ashok and M.R. Douglas, J. High Energy Phys. 01 (2004) 060.

[15] A. Giryavets, S. Kachru, and P. K. Tripathy, J. High Energy Phys. 08 (2004) 002.

[16] J. P. Conlon and F. Quevedo, J. High Energy Phys. 10 (2004) 039.

[17] Z. Lalak, S. Lola, B. A. Ovrut, and G. G. Ross, Nucl. Phys. B434, 675 (1995).

[18] Y. B. Zeldovich, I. Y. Kobzarev, and L. B. Okun, Zh. Eksp. Teor. Fiz. 67, 3 (1974) [Sov. Phys. JETP 40, 1 (1974)].

[19] G. B. Gelmini, M. Gleiser, and E. W. Kolb, Phys. Rev. D 39, 1558 (1989).

[20] J. Preskill, S. P. Trivedi, F. Wilczek, and M. B. Wise, Nucl. Phys. B363, 207 (1991).

[21] A. Vilenkin and E.P.S. Shellard, Cosmic Strings and Other Topological Defects (Cambridge University Press, Cambridge, England, 1994).

[22] A. Vilenkin, Phys. Rev. D 23, 852 (1981).

[23] P. Svrcek and E. Witten, J. High Energy Phys. 06 (2006) 051. 